\title{
The development of a kinematic model to quantify in-shoe foot motion
}

\author{
Chris Bishop ${ }^{1 *}$, Gunther Paul2, Dominic Thewlis ${ }^{1,3}$ \\ From 3rd Congress of the International Foot and Ankle Biomechanics Community \\ Sydney, Australia. 11-13 April 2012
}

\section{Study aim}

To develop a kinematic model to quantify in-shoe foot kinematics during gait.

\section{Methods and material}

Twenty-four participants (mean age $-21.8 \mathrm{yrs} \pm 3.5 \mathrm{yrs}$, height $-1.75 \mathrm{~m} \pm 0.09 \mathrm{~m}$ and body mass $-71.0 \mathrm{~kg} \pm 10.6$ $\mathrm{kg}$ ) were recruited. A marker set consisting of $20 \times 10 \mathrm{~mm}$ markers was developed to track in-shoe joint kinematics [1]. Reliability and accuracy estimates of calibration marker placement on the shoe were determined. To track inshoe foot motion, $12 \mathrm{~mm}$ diameter holes were punched in the shoe upper, with $25 \mathrm{~mm}$ marker wands mounted on the skin through the shoe (Figure 1). The marker set defined a four-segment kinematic model of the foot and ankle (shank, hindfoot, midfoot-forefoot complex and hallux). To define model parameters and moments of inertia, a CT scan was taken of 12 participant's feet. The reconstruction of 3-D bone geometries from two-dimensional grey scale images (DICOM format) was conducted in Simpleware software. Shoe-mounted marker offsets and

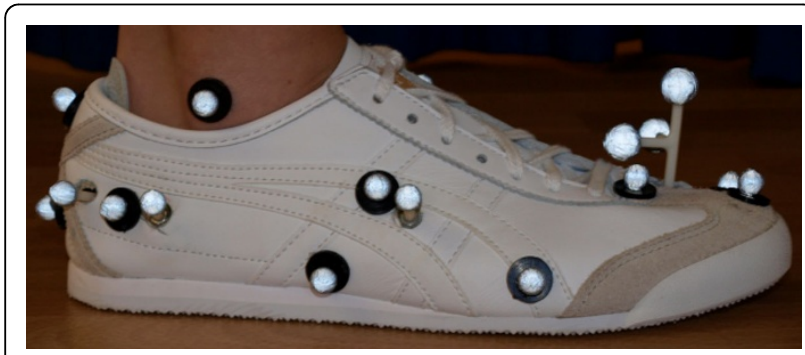

Figure 1 Shod and in-shoe marker set

\footnotetext{
* Correspondence: biscm002@mymail.unisa.edu.au

${ }^{1}$ School of Health Sciences, University of South Australia, Adelaide, South Australia, 5000, Australia
}

Full list of author information is available at the end of the article moments of inertia were inputted to Visual3D. The kinematics of the shoe were described before and after modification to quantify post-modification shoe integrity. The model was deemed sensitive if it detected changes in joint kinematics between conditions that were both statistically significant and greater than the calculated Standard Error of Measurement (SEM) [2].

\section{Results}

The intra-rater $(\mathrm{ICC}=0.68-0.99)$ and inter-rater reliability (ICC $=0.75-0.98)$ of marker placement on the shoe ranged from moderate to excellent. The error of calibration marker placement on the shoe was $<5 \mathrm{~mm}$ compared to skin-mounted markers.

\section{Conclusion}

In conclusion, we present an accurate and reliable kinematic model to describe in-shoe foot kinematics during gait.

\section{Acknowledgements}

ASICS Oceania provided the shoes for the study. Simpleware provided a complimentary software licence used to define model parameters.

\section{Author details}

${ }^{1}$ School of Health Sciences, University of South Australia, Adelaide, South Australia, 5000, Australia. ${ }^{2}$ Mawson Institute, University of South Australia, Adelaide, South Australia, 5041, Australia. ${ }^{3}$ Sansom Institute for Health Research, University of South Australia, Adelaide, South Australia, 5000, Australia.

Published: 10 April 2012

\section{References}

1. Bishop C, et al: The development of a multi-segment kinematic model of footwear. Footwear Sci 2011, 3:S13-S15.

2. Portney LG, Watkins MP: Foundations of Clinical Research: Applications to Practice. Upper Saddle River, NJ: Pearson/Prentice Hall; 2009:xix:892.

doi:10.1186/1757-1146-5-S1-043

Cite this article as: Bishop et al:: The development of a kinematic model to quantify in-shoe foot motion. Journal of Foot and Ankle Research 2012 5(Suppl 1):O43. 